\title{
Patterns of memory: A normative taxonomy of the Wide Range Assessment of Memory and Learning - Second Edition (WRAML-2)*
}

\author{
THOMAS M. ATKINSON, ${ }^{1}$ TIMOTHY R. KONOLD, ${ }^{1}$ AND JOSEPH J. GLUTTING ${ }^{2}$ \\ ${ }^{1}$ Department of Leadership, Foundations, and Policy, University of Virginia Curry School of Education, Charlottesville, Virginia \\ ${ }^{2}$ Department of Education, University of Delaware College of Human Services, Education and Public Policy, Newark, Delaware \\ (Received November 14, 2007; Final Revision June 9, 2008; Accepted June 10, 2008)
}

\begin{abstract}
Memory is arguably the most important function of cognition. When left undetected, memory impairments are linked to life long underachievement and negative social consequences. Given that the construct of memory is multidimensional, the current study examined patterns of multiple indicators associated with memory across individuals ranging in age from 5 to 85 years who had been administered the Wide Range Assessment of Memory and Learning - Second Edition (WRAML-2). Multistage cluster analysis with independent age replications was used to empirically identify normative profiles in a sample of $(n=1172)$ typically developing individuals. This procedure considered how various indicators of memory operate in concert by accounting for the nonlinear multivariate relationships among them. Results supported nine common (or core) profile types that satisfied all formal heuristic and statistical criteria, including complete coverage, satisfactory within-type homogeneity, between-type dissimilarity, and replicability. A summary of the defining characteristics for each profile is provided. (JINS, 2008, 14, 869-877.)
\end{abstract}

Keywords: Cluster analysis, Multivariate analysis, Neuropsychological tests, Data interpretation, Adolescent risk factors, Memory deficits

\section{INTRODUCTION}

Arguably the most important of all cognitive abilities, memory can be defined as the means by which individuals retain and draw on past experiences to use that information in the present (Tulving, 2000). Within the past 40 years, several competing theoretical models of memory have emerged, including the multi-store model (MS; Atkinson \& Shiffrin, 1968), the levels-of-processing model (LOP; Craik \& Lockhart, 1972), working memory models (WM; Baddeley \&

Correspondence and reprint requests to: Thomas M. Atkinson, Memorial Sloan-Kettering Cancer Center, Health Outcomes Research Group, 307 E. 63rd St., 2nd Floor, New York, NY 10065. E-mail: atkinsot@ mskcc.org.

*This research is part of a doctoral dissertation completed at the University of Virginia. An earlier version of this study was presented at the Annual Meeting of the International Neuropsychological Society, Waikoloa, Hawaii, February 9, 2008. The authors wish to thank Xitao Fan, Bruce Gansneder, and Ronald Reeve for their helpful suggestions and Kristen E. Adalian, Jeremy J. Lamica, and Michael P. Stone for their invaluable contributions to the study. There are no financial relationships associated with these results that may reflect a conflict of interest or be perceived to reflect a conflict of interest. This project was not supported by extramural funding.
Hitch, 1974), and the multiple-memory-systems framework (MMS; Tulving, 1972). Regardless of the memory model to which one subscribes, it is clear that impairments in any of the identified indicators of normal memory performance place children and adults at risk in terms of their ability to succeed academically. Deficits have been associated with poor mathematical performance (Holmes \& Adams, 2006), impairment in visual scanning abilities (Schwantes, 1982), problems with attention (Rucklidge, 2006), difficulties in new language/vocabulary acquisition (Alloway et al., 2005a), struggles with categorization (Ashby \& O'Brien, 2005), and reading difficulties (Swanson \& Howard, 2005). When left undiagnosed, memory deficiencies minimally lead to frustration in the child and disengagement from their daily school experiences.

Educators and clinicians have several measurement options at their disposal when attempting to identify potential memory deficits. The Wide Range Assessment of Memory and Learning (WRAML; Sheslow \& Adams, 1990) was recently identified in a survey of clinical neuropsychologists as being one of the most commonly used neuropsychological 
instruments (Rabin et al., 2005). The WRAML is unique in its attempt to meet the perceived clinical need for a measure that is sensitive to changes in memory and learning in children throughout their stages of development, is consistent with current theoretical measures of memory, and includes a sufficient number of educationally relevant and simulating tasks (Sheslow \& Adams, 1990).

Recognizing the lack of consensus on a widely accepted model of memory, the WRAML was influenced by cognitive, neuropsychological, and developmental traditions (Sheslow \& Adams, 1990). This allowed for assessment results to be interpreted within several theoretical frameworks (Mealer et al., 1996), including the MMS and the MS models of memory. More recently, the Wide Range Assessment of Memory and Learning - Second Edition (WRAML-2; Sheslow \& Adams, 2003) entered the market, extending the useful assessment age of the WRAML from 5-17 years to 5-85 years of age. The WRAML-2 is largely an update of the original WRAML that also provides clinicians with a broader menu of optional subtests.

While the assessment of an individual's performance on these measures is an important first step in evaluating potential memory deficits, memory is a multivariate phenomenon that requires joint consideration of how these processes operate in combination rather than in isolation. Although each of the major models of memory referenced above differ with respect to the processes believed to be responsible for memory, all of the systems are complex in the sense that they posit an inter-connected network of mechanisms that operate well in concert among individuals with successful memory capabilities. In the same way, it is likely that individuals will demonstrate different patterns of strengths and weaknesses across measures of different memory functions. As a result, it is important to develop models that integrate the full network of relationships that exist among these abilities to investigate their joint occurrence. The primary purpose of this work is to provide an integrated interpretive framework for evaluating an individual's profile configurations on the six primary subtests of the WRAML-2 that serve as measures of Verbal Memory, Visual Memory, and Attention/Concentration.

In the present study, we use the person-oriented approach of cluster analysis with a large sample of typicallydeveloping individuals ranging from 5 to 85 years of age to identify a normative taxonomy of the most common subtest profiles in the WRAML-2, and then determine whether a mixed clinical sample manifested patterns associated with these profiles. Cluster analysis has been used throughout numerous disciplines to understand the nature of individual differences related to dyslexia (Satz et al., 1985), language (Beitchman et al., 1988; Fegans \& Appelbaum, 1986; Speece et al., 1999), and learning disabilities (Konold et al., 1997; McKinney \& Speece, 1986; Speece et al., 1985). This procedure allowed us to account for the heterogeneous nature of memory functioning and identify homogeneous subgroups that display similar patterns of strengths and weaknesses across these variables that can be used as a normative framework. This is an important first step in evaluating whether a given profile configuration is common or unique.

Profiles are integrated sets of scores that are defined by three elements: level, shape, and scatter (Cronbach \& Gleser, 1953). Treating test scores as integrated profiles provides many benefits. Most notable is that multivariate methods of evaluating profiles account for the full network of relationships among abilities (Sternberg, 1984) and provide greater insight into the nature and complexity of human ability, thereby providing greater diagnostic precision (Glutting et al., 1997). Cross-validation was incorporated into the analysis, and the internal and external validity of the resulting profiles was also investigated.

\section{METHOD}

\section{Participants}

Data from the WRAML-2 standardization sample were used for the primary analysis of identifying normative profile configurations. The 1172 individuals (47\% males) ranged from 5 to 85 years of age $(M=34.83)$, and were distributed as: $5-10$ years $(33.4 \%), 11-20$ years $(16.6 \%), 21-40$ years $(10.2 \%), 41-60$ years $(11.2 \%)$, and over 60 years of age (28.7\%). Test sites were concentrated in four geographic regions of the United States [Northeast (19.0\%), South (34.4\%), Northcentral (22.6\%), and West (24.0\%)]. An effort was made to mirror as closely as possible the 2001 United States Census on the following variables, listed in order of priority: gender, race/ethnicity [African-American (9.6\%), Caucasian $(75.1 \%)$, Hispanic (9.6\%), and Other (4.8\%)], and educational attainment [college and higher (22.4\%), some college (27.6\%), high school graduate $(34.1 \%)$, and less than high school (15.9\%)]. Reported parental education (i.e., the higher level of the mother or father) was used for stratification purposes for participants younger than 17 years of age. Only those that met the census targets in each testing site were selected for standardization; no other exclusion criteria were specified. The study was approved by the University of Virginia Institutional Review Board.

\section{Measure}

The WRAML-2 consists of 17 subtests, each yielding scaled scores ranging from 1 to 19 . Given that the scaling of variables entering a clustering algorithm can influence their relative contributions in the final solution, all measures were standardized to the same $t$-score metric $(M=50$, $S D=10$ ) based on age-specific technical manual conversion tables. Six of these subtests comprise a core group that can be combined to yield three primary indices: Verbal Memory (VBM), Visual Memory (VIM), and Attention/ Concentration (AC). The remaining 11 subtests, including delayed recall tasks, as well as separate Working Memory, Verbal Recognition, and Visual Recognition indices, are optional and can be used to provide supplemental information about the memory function of an individual. The focus 
of the current study was on the six core subtests that are most often administered during individual clinical evaluations. The brief descriptions of the three primary indices and their underlying subtests that follow were obtained from the WRAML-2 administration and technical manual (Sheslow \& Adams, 2003).

Verbal Memory (VBM) focuses on how novel verbal information is processed and recalled and is composed of two core subtests: Story Memory and Verbal Learning. In Story Memory, a participant is read two short stories and is immediately asked to recall as many aspects of the reading passages as possible. Individuals aged 8 years or younger are read stories consisting of 25 and 36 separate aspects, while those 9 or older are read stories containing 36 and 40 aspects, respectively. Verbal Learning involves four repeated trials of an experimenter aurally presenting a participant with a list of simple words, followed by immediate free-recall. The word list consists of 13 items for individuals 8 years or younger and 16 items for those 9 or older.

Visual Memory (VIM) is hypothesized to measure learning and memory of recently presented visual stimuli. The core subtests for this factor are Design Memory and Picture Memory. Design Memory involves the 5-s exposure of a participant to a series of five cards with various geometric forms. After this brief exposure, the individual is asked to draw all aspects of the image that they are able to recall. In Picture Memory, participants are asked to identify changes that have been made to four separate common, yet complex visual images after a 10-s exposure to each image.

Attention/Concentration (AC) measures the same named abilities through the use of two core subtests: Finger Windows and Number Letter. The Finger Windows subtest presents participants with a vertically resting card containing asymmetrically located holes. In each trial, the examiner demonstrates a pattern of placing a pencil eraser in a sequence of holes and then asks the individual to duplicate the sequence by placing their finger in each hole according to the order of presentation. The length of the sequence of holes increases after each trial. The Number Letter subtest is similar to a digit span task; participants are aurally presented with sequences of alternating numbers and letters and then asked to recall this information in the order it was presented.

Psychometric properties of the six primary subtests are well documented and favorable (Sheslow \& Adams, 2003). Internal consistency measures were in the high to excellent range (.86-.93) for the majority of subtests. Confirmatory factor analyses (CFA) demonstrated that, consistent with the hypothesized framework, a three-factor model best represents the six core subtests. Multi-group structural analyses provided evidence that the three-factor solution was invariant across groups reflecting gender, ethnicity, age, and level of education. In addition, various subtests of the WRAML-2 demonstrated an acceptable degree of correlation with other instruments designed for the measurement of memory, including the Wechsler Memory Scale-III ( $r=.60)$, Children's Memory Scale $(r=.49)$, Test of Memory and Learning $(r=.69)$, the California Verbal Learning Test $(r=.64)$, and the California Verbal Learning Test-II $(r=.68)$.

\section{Clustering Strategy}

The clustering strategy we adopted was similar to the one used elsewhere for identifying normative profiles (Glutting \& McDermott, 1990; Glutting et al., 1997; Konold et al., 1997), as described in McDermott (1998). This procedure involved three steps. In the first step, Ward's (1963) hierarchical-agglomerative procedure was performed on a Euclidean distance matrix that is sensitive to level, shape, and scatter. Ward's method has been shown to outperform alternative methods in terms of minimizing profile overlap (Bayne et al., 1980) and to be the most efficient means by which to recover known taxonomic structure in a population exhibiting variation (Kuiper \& Fisher, 1975). In this first step, the total sample $(n=1172)$ was divided into age level partitions to form five blocks, consistent with the predefined age groups of the WRAML-2 (i.e., 5-10, 11-20, 21-40, 41-60, and over 61; Sheslow \& Adams, 2003), thereby allowing for an internal age based replication analysis. Decisions regarding the number of clusters to retain within each of the five samples were based on several indices: Pseudo- $F$ (Calinski \& Harabasz, 1985), pseudo $t^{2}$ (Duda \& Hart, 1973), $R^{2}$, inspection of the agglomeration index for each subgroup as well as the agglomeration history within each subgroup, the coherence of the resulting clusters, and the degree of replicability of clusters across subgroups (Crockett et al., 2006). This step also used a "trim" procedure that removed a maximum of $2 \%$ of the outlier cases from consideration in the analysis (McDermott, 1998).

Information from the clusters identified in step 1 was pooled to form an overall similarity matrix that was used for step 2. Thus, step 2 clustering began with a proximity matrix whose diagonal elements held error sums of squares (ESS) statistic values for respective step 1 clusters, with off-diagonal elements corresponding to potential ESSs for merging each pair of first-stage clusters. Ward's method was used on the resulting similarity matrix from step 1 to assess the extent to which cluster profiles from subsamples of the data matched those found for the total sample (i.e., replication). Each of the aforementioned statistical indices was again considered when determining how many clusters to retain at step 2. Steps 1 and 2 led to the identification of nine clusters. Clusters 8 and 9 yielded replication rates of $60 \%$ and $80 \%$, respectively. The remaining seven clusters demonstrated replication rates of $100 \%$ (Table 1). The replication rate of $80 \%$ indicates that profile nine was also identified in four of the five subsamples, whereas, profiles demonstrating $100 \%$ replication rates were found to emerge in all five subsamples of step 1.

Group centroids from the step 2 solution served as starting seeds for the stage 3 iterative partitioning analysis conducted using $K$-means passes. $K$-means cluster analysis makes use of an iterative procedure where individuals are 
Table 1. Prevalence and psychometric properties of the WRAML-2 subtest taxonomy

\begin{tabular}{lcccc}
\hline \hline $\begin{array}{c}\text { Cluster no. } \\
(n=1172)\end{array}$ & $\begin{array}{c}\text { \% Population } \\
\text { prevalence }\end{array}$ & $\begin{array}{c}\text { Internal profile } \\
\text { cohesion }\end{array}$ & $\begin{array}{c}\text { External } \\
\text { isolation }\end{array}$ & $\begin{array}{c}\text { Independent replications } \\
\text { across 5 age blocks }\end{array}$ \\
\hline 1 & 8.9 & 0.75 & 1.00 & 100 \\
2 & 10.8 & 0.68 & -0.16 & 100 \\
3 & 11.2 & 0.76 & 0.43 & 100 \\
4 & 13.3 & 0.74 & -0.01 & 100 \\
5 & 12.0 & 0.71 & 0.47 & 100 \\
6 & 11.4 & 0.79 & 0.47 & 100 \\
7 & 13.3 & 0.77 & 0.42 & 60 \\
8 & 9.1 & 0.75 & 0.54 & 80 \\
9 & 10.0 & 0.78 & 0.27 & 93.33 \\
Average & 100.0 & 0.75 & 0.38 & \\
\hline \hline
\end{tabular}

assigned to core subgroup membership based upon their smallest Euclidean distance to each subsequent cluster centroid (Eng et al., 2000; Jones et al., 2006).This third step was necessary because hierarchical-agglomerative procedures (steps 1 and 2) do not allow subjects to shift clusters after their original assignment, despite the fact that they may fit better in a different profile later in the solution. By contrast, iterative partitioning procedures allow subjects to migrate to neighboring clusters, following identification of the number of suspected clusters (steps 1 and 2), and generally result in tighter solutions.

\section{RESULTS}

Mean profile configurations for the resulting nine cluster solution are presented in Table 2. The nine profiles represent the natural variation of individual memory disparity and are typical of what we would expect among the general population.
Table 1 provides other psychometric properties for each profile. The final cluster solution from step 3 was required to retain the dual properties of internal cohesion and external isolation (Aldenderfer \& Blashfield, 1984). Both internal cohesion and external isolation address the issue of internal validity. Internal cohesion refers to the tightness of a cluster, or the closeness of objects around the cluster centroid. External isolation refers to the distance between clusters in multivariate space. Thus, subjects within a given cluster should be similar to one another, whereas clusters composed of homogeneous individuals should be distinct from one another. The average $H$ coefficient (Tryon \& Bailey, 1970) across profiles satisfied a priori expectations for internal cluster cohesion $\geq .60$ (Average $H=.75$ ), thereby, providing evidence in support of homogeneous within-cluster representation. In addition, the average $r_{p}$ (Cattell, 1949) across profiles also satisfied a priori expectation in support of external isolation $<.40$ (Average $r_{p}=.38$ ).

Table 2. Mean WRAML-2 subtest and factor score patterns by profile type

\begin{tabular}{|c|c|c|c|c|c|c|c|c|c|c|}
\hline \multirow{3}{*}{$\begin{array}{l}\text { Profile } \\
\text { type }\end{array}$} & \multicolumn{6}{|c|}{ Clustered subtest variables } & \multicolumn{3}{|c|}{ Factor scores } & \multirow[t]{3}{*}{ Profile name and description } \\
\hline & \multicolumn{2}{|c|}{ VBM } & \multicolumn{2}{|c|}{ VIM } & \multicolumn{2}{|c|}{$\mathrm{AC}$} & \multirow[b]{2}{*}{ VBM } & \multirow[b]{2}{*}{ VIM } & \multirow[b]{2}{*}{$\mathrm{AC}$} & \\
\hline & $\mathrm{SM}$ & VL & $\mathrm{DM}$ & PM & FW & NL & & & & \\
\hline 1 & 62.45 & 60.47 & 58.93 & 58.73 & 58.43 & 57.46 & 61.46 & 58.83 & 57.95 & Above Average Memory Skills \\
\hline 2 & 52.33 & 53.38 & 50.95 & 46.71 & 62.47 & 60.92 & 52.86 & 48.83 & 61.70 & Above Average Attention/Concentration \\
\hline 3 & 57.33 & 55.95 & 60.46 & 54.40 & 46.11 & 46.72 & 56.64 & 57.43 & 46.42 & Above Average Verbal and Visual Memory \\
\hline 4 & 47.85 & 45.62 & 56.06 & 58.39 & 49.05 & 54.05 & 46.74 & 57.23 & 51.55 & Above Average Visual Memory \\
\hline 5 & 57.05 & 57.03 & 41.85 & 50.40 & 50.61 & 49.39 & 57.04 & 46.13 & 50.00 & $\begin{array}{l}\text { Above Average Verbal Memory with } \\
\text { Depressed Design Memory }\end{array}$ \\
\hline 6 & 44.42 & 56.88 & 53.60 & 45.54 & 55.86 & 46.94 & 50.65 & 49.57 & 51.40 & Variable Memory Skills \\
\hline 7 & 46.15 & 45.01 & 44.53 & 42.20 & 47.09 & 55.19 & 45.58 & 43.37 & 51.14 & $\begin{array}{l}\text { Slightly Below Average Memory with } \\
\text { Elevated Number Letter Skills }\end{array}$ \\
\hline 8 & 45.64 & 45.50 & 47.00 & 54.25 & 41.24 & 39.26 & 45.57 & 50.63 & 40.25 & $\begin{array}{l}\text { Slightly Below Average Memory with } \\
\text { Elevated Picture Memory Skills }\end{array}$ \\
\hline 9 & 38.25 & 38.21 & 40.47 & 38.28 & 43.33 & 39.84 & 38.23 & 39.38 & 41.59 & Below Average Memory Skills \\
\hline
\end{tabular}

Note. SM, Story Memory; VL, Verbal Learning; DM, Design Memory; PM, Picture Memory; FW, Finger Windows; NL, Numbers-Letters; VBM, Verbal Memory Index; VIM, Visual Memory Index; AC, Attention/Concentration Index. 


\section{Profile Descriptions}

In the descriptions that follow, we present a synthesis of information reported in Tables 2 and 3 pertaining to the group averages demonstrated by these profiles and associated demographic features. We have chosen to name each group according to that feature of functioning that appears to distinguish it from the other groups. Thus, the names below reflect a distinguishing feature and by implication also reflect average functioning in the other domains assessed. Profile names are provided to highlight relative strengths and weaknesses that were found through empirical methods as a way of differentiating among individuals that are not known to be at-risk. The summaries below also take into consideration the demographic factors of ethnicity, education level, and age. For each demographic category, observed percentages were compared with expected values based on the composition of the overall sample through methods of standard error of proportional differences tests. An alpha level of .006 (.05/9) was used to adjust for multiple comparisons and reduce the likelihood of Type I error. Observed percentages of males and females did not statistically differ from expected percentages in any of the nine identified profiles.

\section{Above average memory skills ( prevalence $=12.0 \%)$}

This cluster demonstrated a relatively flat above average profile across each of the six memory indicators. Fewer than half of the anticipated African-American and Hispanic participants were present in this profile. There were over two times more individuals with college and higher levels of education than expected, and a significantly fewer than expected number of individuals reported that high school graduation or less than high school was their highest education level.

\section{Above average attention/concentration (prevalence $=8.9 \%)$}

This pattern demonstrated the lowest rate of prevalence. Individuals in Profile 2 exhibited above average scores on the subtests that comprise the Attention/Concentration factor (i.e., Finger Windows and Number Letter). Half of the expected African-American participants and less than a quarter of the anticipated Hispanics were found in this profile. While significantly fewer members of this profile were members of the "high school graduate" and "less than high school" groups, a greater than expected proportion of participants had a beyond college level education. In addition, there were significantly more members of the 21 - to 40 - and 41 to 60-year-old age groups.

\section{Above average verbal and visual memory (prevalence $=10.0 \%)$}

Members had above average scores on the four subtests (i.e., Story Memory, Verbal Learning, Design Memory, and

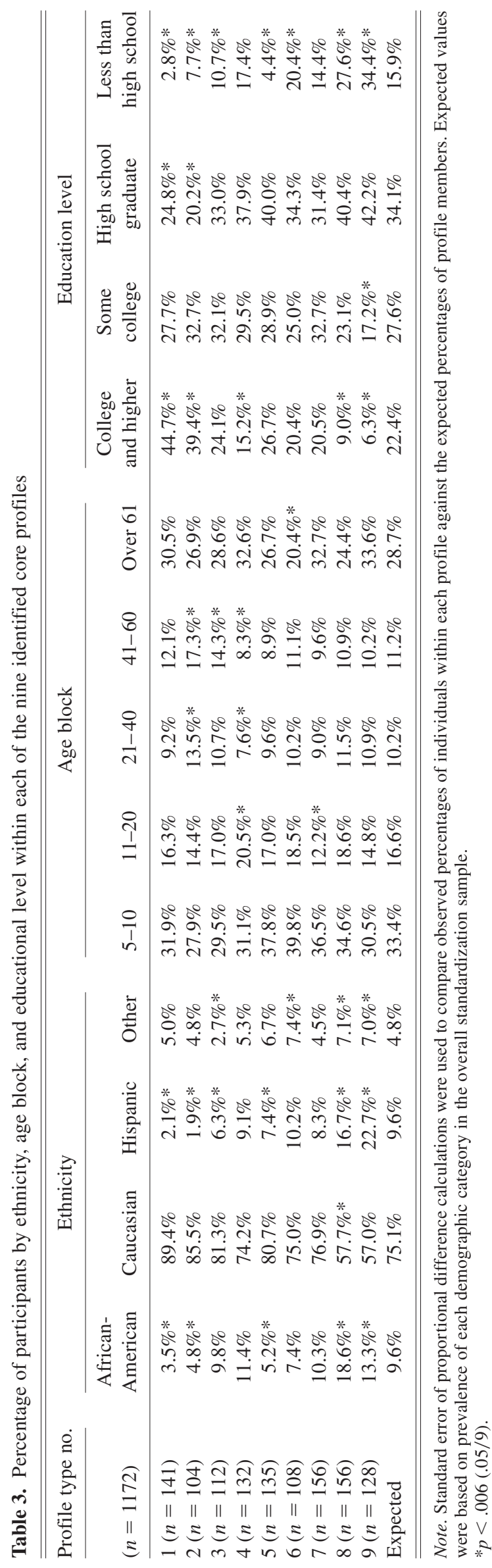


Picture Memory) underlying the Verbal and Visual Memory factors. A smaller concentration of Hispanics and participants in the "other" ethnicity category were found in this profile than was anticipated. Fewer than expected number of participants in this profile reported having attained less than a high school diploma, and a significantly higher proportion of participants were found in the 41- to 60-year-old age block relative to the overall sample.

\section{Above average visual memory (prevalence $=11.2 \%$ )}

Visual Memory was elevated among members of this profile as evidenced by somewhat above average performance on Design Memory and Picture Memory. Significantly fewer individuals obtained a college or higher level of education. There were more members of the 11- to 20-year-old age group, and fewer members of the 21- to 60-year-old age groups than expected. Ethnicity composition met expectations in this profile.

\section{Above average verbal memory with depressed design memory \\ (prevalence $=11.4 \%)$}

Individuals in Profile 5 demonstrated higher scores on the Story Memory and Verbal Learning (measures of Verbal Memory), and performed below average on Design Memory. This profile consisted of a lower concentration of African-American and Hispanic participants than was projected. Age groups were represented in proportion to the overall sample. However, significantly fewer individuals with this profile reported not having attained a high school diploma.

\section{Variable memory skills (prevalence $=9.1 \%$ )}

Members of this profile were somewhat above average on one index of each of the three factors (Verbal Learning, Design Memory, and Finger Windows) and somewhat below average on the remaining index of the three factors (Story Memory, Picture Memory, and Number Letter). A higher than expected proportion of participants in the "other" ethnicity category was found in this profile. A large percentage of participants reported having less than a high school education level (20.4\%) when compared with the composition of the standardization sample, and there was a less than anticipated concentration of participants older than 61 years of age in this profile.

\section{Slightly below average memory with elevated number letter skills \\ ( prevalence $=13.3 \%$ )}

Ethnicity and education level distributions within this profile were within expectation; however, fewer individuals were members of the 11- to 20-year-old age group.

\author{
8. Slightly below average memory with \\ elevated picture memory skills \\ ( prevalence $=13.3 \%)$
}

Significantly more African-Americans, Hispanics and members of the "other" ethnicity category were found in this profile, while there was a fewer than expected percentage of Caucasians. Less than half of the expected percentage of participants in this profile had attained at least a college degree, while a greater proportion had yet to attain a high school diploma. Age distributions within this profile were consistent with expected distributions.

\section{Below average memory skills ( prevalence $=10.8 \%)$}

This profile configuration was representative of generally below average performance on measures of memory. A larger proportion of African-Americans, Hispanics, and those in the "other" ethnicity category were found in this profile than was expected. More than twice the expected proportion of participants with this profile attained less than a high school diploma, while significantly less had attended college or at least completed a college degree. Age group distributions were consistent with expectations.

\section{Profile Stability}

Profile stability was examined with $n=142$ participants, the majority of which were included in the original normative standardization sample and were also retested following a period of time ( $M=73.5$ days, $S D=63.9$ days $)$. To objectively compare the normative core profiles obtained from the standardization sample with those in the retested sample, it was necessary to select a statistical strategy for evaluating pattern similarity. The accuracy of two procedures for evaluating pattern similarity was first examined within the normative sample $(n=1172)$ to determine which approach yielded the highest classification rates when cluster memberships were known. Cattell's $r_{p}$ technique (1949) revealed pattern similarity return rates of $84.4 \%$, whereas the distance measure $\left(D^{2} ; 1952\right)$ of Osgood and Suci (1952) yielded somewhat more favorable return rates of $97.7 \%$. Consequently, Osgood and Suci's $D^{2}$ was used with the retest sample $(n=142)$ to gauge profile stability.

Sixty-five of the 142 participants $(65 / 142=45.8 \%)$ were classified to the same profile configuration based on their initial testing and subsequent follow-up. This stability estimate represents a material increase over what would be expected by chance $(12.4 \%)$ assignment across the nine profiles. In addition, it is important to note that there was not a statistically significant difference in the number of re-test days between participants that were found to have the same profile configurations at both testing occasions $(M$ days $=81.06)$ and those that $\operatorname{did} \operatorname{not}(M$ days $=67.05)$; $t(140)=-1.30 ; p=.19$. 


\section{DISCUSSION}

Despite the ongoing debate as to which theoretical model of memory is most representative of the underlying mechanisms of the construct, the importance of this cognitive ability in everyday function is unquestionable. When left undetected, memory impairments are linked to lifelong underachievement and negative social consequences (Alloway et al., 2005b; Callu et al., 2005; Swanson \& Howard, 2005). The WRAML-2 was designed to be a comprehensive tool to be used in the detection of clinically meaningful deficiencies in memory ability throughout the lifespan (Sheslow \& Adams, 2003). The purpose of the present study was to identify a normative taxonomy of profiles likely to be found among typically developing individuals. Nine normative patterns of memory were identified through a multivariate model that simultaneously considered multiple measures of memory, reflecting a range of underlying constructs related to verbal, visual, and attention/concentration abilities. Profiles were defined in terms of patterns of relative strengths and weaknesses on these assessments.

The six subtests of the WRAML-2 core battery, on which the profiles were obtained, have been shown to measure three components of memory: verbal memory, visual memory, and attention/concentration (Sheslow \& Adams, 2003). As such, the normative profiles presented here may be particularly useful for differentiating between normal and other clinical groups than were the focus of the current investigation. For example, a study of memory performance in patients experiencing severe depression demonstrated differences in performance on measures of verbal memory, visual memory, and attention/concentration (Hihn et al., 2006). Morse and Rice (2005) treated verbal memory, visual memory, and attention and concentration as separate systems in an investigation of memory in postmenopausal women. Patient outcomes on measures of verbal memory, visual memory, and attention/concentration were among several dimensions examined in a study of the relationship between educational attainment and cognitive performance in a group of individuals with subarachnoid hemorrhage (Nakhutina, 2006). Examination of profile configurations of these, and other known clinical groups, would be important next steps for extending the clinical utility of profile analyses as related to memory. In addition, it may be useful to revisit this analysis and include the WRAML-2 optional subtests from the three memory components, as well as the supplemental areas of working memory, verbal recognition, and visual recognition to capture a somewhat more global understanding of memory and learning in an individual.

It should be pointed out that while the present study may appear to indicate that individuals belonging to a particular demographic (e.g., age group) may specifically exhibit higher performance than others in the sample, caution should be used when interpreting these relationships. While such comparisons could prove as useful for clinicians, future research is needed before making specific comparisons between demographic groups on any of the identified WRAML-2 profiles. This is particularly true when making profile comparisons based upon ethnicity, given the small sample sizes for the African American and Hispanic groups in relation to the Caucasian participants.

To meet analysis requirements and to remain consistent with methodology during test standardization, the original 15 age groups (i.e., ages 5, 6, 7, 8, 9-10, 11-13, 14-17, $18-24,25-39,40-54,55-64,65-69,70-74,75-79$, and over 80) were collapsed into five blocks. Combining these age groups may obscure the ability to detect differences in profiles throughout the lifespan. To recover potentially useful information related to age differences, a larger sample should be collected with at least 150-200 participants in each age group. It should be pointed out that sample size did not impact the overall magnitude of the cluster analysis in this study, as all internal cohesion, external isolation, and a priori replication requirements were met.

The present results may assist clinicians during the process of instrument selection in memory assessment. Despite somewhat frequent use of the original WRAML (Rabin et al., 2005) the WRAML-2 has been sparsely mentioned in the current literature (Hall, 2006, 2007; Hartman, 2007; Shaver, 2005). Consequentially, it is important to establish the psychometric qualities of this instrument to help clinicians determine whether this test is a viable alternative for the existing methods of memory assessment. As opposed to some of the more established instruments (i.e., CFT, CVLT-C, CMS), the WRAML-2 is currently the only measure of memory that can be administered across the lifespan (Sheslow \& Adams, 2003). To compete with the features of other memory assessment instruments, as well as provide clinicians with desired supplemental information, several optional subtests are available to assess delayed recall, verbal and visual recognition, as well as working memory. In addition, results of the present study were consistent with a cluster analysis of the CVLT-C (Donders, 1999). To further compare across memory assessment instruments, it would be helpful to identify common profiles in each of these tools and determine sensitivity to the detection of clinical impairment.

The obtained normative taxonomy of profiles provides clinicians with a useful tool for evaluating whether observed score patterns on the WRAML-2 are deviant, or simply reflect normal subtest score variation. Through direct comparisons with results from the present study, individuals that would be typically identified as having memory or learning deficits based upon normative information may, in fact, be scoring within a given normal profile. It is suggested that future test standardizations incorporate cluster analysis to provide clinicians with additional pieces of information for identifying potential deficiencies in abilities.

Finally, it is important to provide some additional context for the obtained profile stability analysis conducted on the 142 test-retest participants. Although we are encouraged by the $45.8 \%$ return rate that was materially greater than what would be expected by chance (i.e., 12.4\%), the fact remains that over half of the participants migrated from 
their original profile type on subsequent evaluation. Unfortunately, the assessment of memory and learning does not lend itself well to score consistency in a longitudinal sense. Sheslow and Adams (2003) discussed this point in the WRAML-2 technical manual:

With memory tests, there is an inherent problem with measuring stability in that the test itself is predicated on the retention of specific material presented at the time of testing. The giving of the test a second time presents more opportunity for learning and, therefore, can contaminate the original purpose of the testing, thus spoiling the stability measurement. With measurements of reasoning, achievement, or other cognitive abilities, this effect is not as severe because the intent of these measures is not learning per se but the assessment of what has been learned outside the test situation and applied in some way to the test content. (p. 100).

Test-retest estimates for the six WRAML-2 subtests that served as the basis for cluster formation ranged from .53 to .78 in the standardization sample (Sheslow \& Adams, 2003). In our retest sample, the majority of participant's scores across the six measures increased from the first to second testing $(56 \%)$, while others decreased $(23.35 \%)$ or remained the same $(18.65 \%)$. Consequently, clinicians are encouraged to evaluate individual profile configurations, relative to the normative subtypes identified here, based on first evaluations to capitalize on the novelty of the client experience with respect to exam content.

\section{REFERENCES}

Aldenderfer, M.S. \& Blashfield, R.K. (1984). Cluster Analysis. Beverly Hills, CA: Sage Press.

Alloway, T.P., Gathercole, S.E., \& Adams, A.-M. (2005a). Working memory and phonological awareness as predictors of progress towards early learning goals at school entry. British Journal of Developmental Psychology, 23, 417-426.

Alloway, T.P., Gathercole, S.E., \& Adams, A.-M. (2005b). Working memory abilities in children with special educational needs. Educational and Child Psychology, 22, 55-67.

Ashby, F.G. \& O'Brien, J.B. (2005). Category learning and multiple memory systems. Trends in Cognitive Sciences, 9, 83-89.

Atkinson, R.C. \& Shiffrin, R.M. (1968). Human memory: A proposed system and its control processes. In K.W. Spence \& J.T. Spence (Eds.), The Psychology of Learning and Motivation: Vol. 2. Advances in Research and Theory. New York: Academic Press.

Baddeley, A.D. \& Hitch, G.J. (1974). Working memory. In G.A. Bower (Ed.), The Psychology of Learning and Motivation. New York: Academic Press.

Bayne, R., Beauchamp, J., Begovich, C., \& Kane, V.E. (1980). Monte Carlo comparisons of selected clustering procedures. Pattern Recognition, 12, 51-62.

Beitchman, J.H., Hood, J., Rochon, J., Mantini, T., \& Majumdar, S. (1988). Empirical classification of speech/language impairment in children: Identification of speech/language categories. Journal of the American Academy of Child and Adolescent Psychiatry, 28, 112-117.
Calinski, T. \& Harabasz, J. (1985). A dendrite model for cluster analysis. Communications in Statistics, 3, 1-27.

Callu, D., Giannopulu, I., \& Escolano, S. (2005). Smooth pursuit eye movements are associated with phonological awareness in preschool children. Brain and Cognition, 58, 217-225.

Cattell, R.B. (1949). $r_{p}$ and other coefficients of pattern similarity. Psychometrika, 14, 279-298.

Craik, F.I.M. \& Lockhart, R.S. (1972). Levels of processing: A framework for memory research. Journal of Verbal Learning and Verbal Behavior, 11, 671-684.

Crockett, L.J., Moilanen, K.L., Raffaelli, M., \& Randall, B.A. (2006). Psychological profiles and adolescent adjustment: A person-centered approach. Development and Psychopathology, 18, 195-214.

Cronbach, L. \& Gleser, G. (1953). Assessing similarity between profiles. Psychological Bulletin, 50, 456-473.

Donders, J. (1999). Cluster subtypes in the standardization sample of the California Verbal Learning Test - Children's Version. Developmental Neuropsychology, 16, 163-175.

Duda, R.O. \& Hart, P.E. (1973). Pattern Classification and Scene Analysis. New York: Wiley.

Eng, W., Heimberg, R.G., Coles, M.E., Schneier, F.R., \& Liebowitz, M.R. (2000). An empirical approach to subtype identification in individuals with social phobia. Psychological Medicine, 30, 1345-1357.

Fegans, L. \& Appelbaum, M.I. (1986). Validation of language subtypes in learning disabled children. Journal of Educational Psychology, 78, 358-364.

Glutting, J.J. \& McDermott, P.A. (1990). Patterns and prevalence of core profile types in the WPPSI standardization sample. School Psychology Review, 19, 471-491.

Glutting, J.J., McDermott, P.A., \& Konold, T.R. (1997). Ontology, structure and diagnostic benefits of a normative subtest taxonomy from the WISC-III standardization sample. In D.P. Flanagan, J.L. Genshaft, \& P.L. Harrison (Eds.), Contemporary Intellectual Assessment: Theories, Tests, and Issues (pp. 349372). New York: Guilford Press.

Hall, S.J. (2007). Comparison between memory test results among persons with Alzheimer's disease. Dissertation Abstracts International: Section B: The Sciences and Engineering, 67, 4708.

Hall, T.A. (2006). The Everyday Memory Survey: Development and psychometric analysis. Dissertation Abstracts International: Section B: The Sciences and Engineering, 66, 3993.

Hartman, D.E. (2007). Test review: Wide Range Assessment of Memory and Learning-2 (WRAML-2): WRedesigned and WReally improved. Applied Neuropsychology, 14, 138-140.

Hihn, H., Baune, B.T., \& Michael, N. (2006). Memory performance in severely depressed patients treated by electroconvulsive therapy. The Journal of ECT, 22, 189-195.

Holmes, J. \& Adams, J.W. (2006). Working memory and children's mathematical skills: Implications for mathematical development and mathematics curricula. Educational Psychology, 26, 339-366.

Jones, P.R., Laufgraben, J.L., \& Morris, N. (2006). Developing an empirically based typology of attitudes of entering students toward participation in learning communities. Assessment \& Evaluation in Higher Education, 31, 249-265.

Konold, T.R., Glutting, J.J., \& McDermott, P.A. (1997). The development and applied utility of a normative aptitude-assessment taxonomy for the Woodcock-Johnson Psycho-Educational Battery-Revised. The Journal of Special Education, 31, 212-232. 
Kuiper, F.K. \& Fisher, L. (1975). 391: A Monte Carlo comparison of six clustering procedures. Biometrics, 31, 777-783.

McDermott, P.A. (1998). MEG: Megacluster analytic strategy for multistage hierarchical grouping with relocations and replications. Educational and Psychological Measurement, 58, 677-686.

McKinney, J.D. \& Speece, D.L. (1986). Academic consequences and longitudinal stability of behavioral subtypes of learning disabled children. Journal of Educational Psychology, 78, 365-372.

Mealer, C., Morgan, S., \& Luscomb, R. (1996). Cognitive functioning of ADHD and non-ADHD boys on the WISC-III and WRAML: An analysis within a memory model. Journal of Attention Disorders, 1, 133-145.

Morse, C.A. \& Rice, K. (2005). Memory after menopause: Preliminary considerations of hormone influence on cognitive functioning. Archives of Women's Mental Health, 8, 155-162.

Nakhutina, L. (2006). The effect of educational attainment on cognitive performance and recovery in patients with subarachnoid hemorrhage (SAH). Dissertation Abstracts International: Section B: The Sciences and Engineering, 67, 1743.

Osgood, C.E. \& Suci, G.J. (1952). A measure of relation determined by both mean difference and profile information. Psychological Bulletin, 49, 251-262.

Rabin, L.A., Barr, W.B., \& Burton, L.A. (2005). Assessment practices of clinical neuropsychologists in the United States and Canada: A survey of INS, NAN, and APA Division 40 members. Archives of Clinical Neuropsychology, 20, 33-65.

Rucklidge, J.J. (2006). Gender differences in neuropsychological functioning of New Zealand adolescents with and without attention deficit hyperactivity disorder. International Journal of Disability, Development, and Education, 53, 47-66.

Satz, P., Morris, R., \& Fletcher, J.M. (1985). Hypotheses, subtypes and individual differences in dyslexia: Some reflections. In D.B. Gray \& J.F. Kavanagh (Eds.), Biobehavioral Measures of Dyslexia. Parkton, MD: York Press.

Schwantes, F.M. (1982). Developmental differences in cognitive scanning and encoding of visual information. Journal of Experimental Child Psychology, 34, 301-310.
Shaver, G.W. (2005). Distinguishing simulated malingerers from head injured patients and controls on the Wide Range Assessment of Memory and Learning - Second Edition. Dissertation Abstracts International: Section B: The Sciences and Engineering, 65, 6674 .

Sheslow, D. \& Adams, W. (1990). Wide Range Assessment of Memory and Learning. Wilmington, DE: Jastak.

Sheslow, D. \& Adams, W. (2003). Wide Range Assessment of Memory and Learning - Second Edition: Administration and Technical Manual. Wilmington, DE: Wide Range, Inc.

Speece, D.L., McKiney, J.D., \& Appelbaum, M.I. (1985). Classification and validation of behavioral subtypes of learningdisabled children. Journal of Educational Psychology, 77, $67-77$.

Speece, D.L., Roth, F.P., Cooper, D.H., \& LaPaz, S.D. (1999). The relevance of oral language skills to early literacy: A multivariate analysis. Applied Psycholinguistics, 20, 167-190.

Sternberg, R.J. (1984). The Kaufman Assessment Battery for Children: An information-processing analysis and critique. Journal of Special Education, 18, 269-278.

Swanson, H.L. \& Howard, C.B. (2005). Children with reading disabilities: Does dynamic assessment help in the classification? Learning Disability Quarterly, 28, 17-34.

Tryon, R.C. \& Bailey, D.E. (1970). Cluster Analysis. New York, NY: McGraw-Hill.

Tulving, E. (1972). Episodic and semantic memory. In E. Tulving \& W. Donaldson (Eds.), Organization of Memory. New York: Academic Press.

Tulving, E. (2000). Memory: An overview. In A.E. Kazdin (Ed.), Encyclopedia of Psychology (Vol. 5, pp. 161-162). Washington, DC: American Psychological Association.

Ward, J.H., Jr. (1963). Hierarchical grouping to optimize an objective function. Journal of the American Statistical Association, $58,236-244$. 